\title{
SPICES: A Mission Concept to Characterize Long Period Planets from Giants to Super-Earths
}

\author{
Anthony Boccaletti ${ }^{1}$, Anne-Lise Maire ${ }^{1}$, Raphaël Galicher ${ }^{2}$, Pierre
} Baudoz $^{1}$, Dimitri Mawet ${ }^{3}$, John Trauger ${ }^{4}$, Jean Schneider ${ }^{5}$, Wes

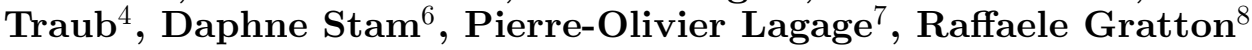
and the SPICES team

\author{
${ }^{1}$ LESIA, Obs. Paris/CNRS/Univ. Paris 6/Univ. Paris 7, 92195 Meudon, France \\ email: anthony.boccaletti@obspm.fr \\ ${ }^{2}$ Herzberg Institute of Astrophysics, NRCC, Victoria, BC V9E 2E7, Canada \\ ${ }^{3}$ European Southern Observatory, Casilla 19001, Santiago 19, Chile \\ ${ }^{4}$ JPL, CalTech, Pasadena, CA 91109, USA \\ ${ }^{5}$ LUTH, Obs. Paris/CNRS/Univ. Paris 7, 92195 Meudon, France \\ ${ }^{6}$ SRON, Utrecht, The Netherlands \\ ${ }^{7} \mathrm{SAp} / \mathrm{CEA}$, Saclay, France \\ ${ }^{8}$ INAF, Padova Observatory, Padova, Italy
}

\begin{abstract}
SPICES (Spectro-Polarimetric Imaging and Characterization of Exoplanetary Systems) was proposed in 2010 for a five-year M-class mission in the context of ESA Cosmic Vision. Its purpose is to image and characterize long-period extrasolar planets located at several AUs (0.5-10 AU) from nearby stars $(<25 \mathrm{pc})$ with masses ranging from a few Jupiter masses down to super-Earths $\left(\sim 2\right.$ Earth radii, $\left.\sim 10 \mathrm{M}_{\oplus}\right)$, possibly habitable. In addition, circumstellar disks as faint as a few times the zodiacal light in the Solar System can be studied. SPICES is based on a $1.5-\mathrm{m}$ off-axis telescope and can perform spectro-polarimetric measurements in the visible $(450-900 \mathrm{~nm})$ at a spectral resolution of about 40. This paper summarizes the top science program and the choices made to conceive the instrument. The performance is illustrated for a few emblematic cases.
\end{abstract}

Keywords. instrumentation: high angular resolution, planetary systems

\section{Science program}

SPICES is an evolution of the former SEE-COAST proposal (Schneider et al. 2009) and belongs to the category of so-called small coronagraphic telescopes derived from the Terrestrial Planet Finder concept (Levine et al. 2009). It is a direct imaging mission designed to achieve very high contrasts in order to characterize exoplanetary systems previously identified by other instruments/techniques (radial velocity, astrometry, direct imaging). This challenging goal clearly requires a specific instrumentation, which is not available on any other present or future facility and must be optimized for a very specific task. Typical star/planet brightness ratios in reflected light are in the range of $10^{8}$ to $10^{10}$ at less than 1 arcsec. SPICES combines several techniques for high contrast imaging in a single instrument designed to maximize the astrophysical return while reducing risks. A more precise description of the proposal can be found in Boccaletti et al. (2012) while detailed simulations are presented in Maire et al. (2012). We here extract a few important results from these two reference papers.

The primary objective of SPICES is to characterize the atmospheres and possibly surfaces of various types of planets: gaseous and iced giants as well as super-Earths. For 

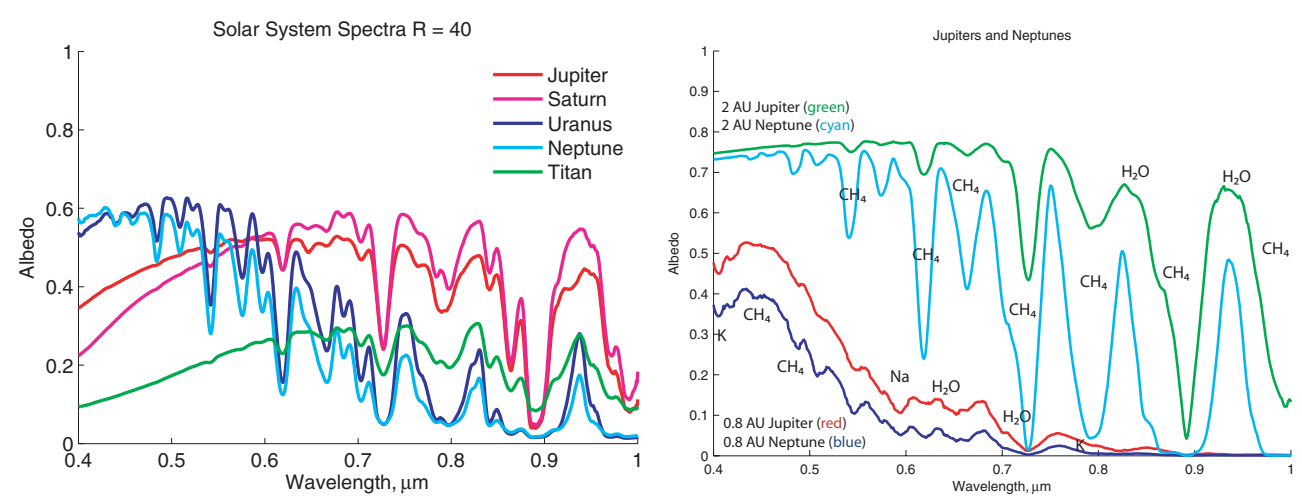

Figure 1. Right: Albedo of some planets in the Solar System (Karkoschka 1994) smoothed to SPICES resolution, $\mathrm{R}=40$. Left: Albedo of model Jupiter and Neptune planets from Cahoy et al. (2010) at separations of $0.8 \mathrm{AU}$ (red and dark blue, too warm for clouds) and $2 \mathrm{AU}$ (green and cyan, just cold enough for water clouds) smoothed to SPICES resolution, $\mathrm{R}=40$.

that purpose, SPICES combines direct imaging with spectro-polarimetry in the visible (450 - $900 \mathrm{~nm}$ ) and measures the total flux F and the linearly polarized fluxes Q and U, all as functions of the wavelength $\lambda$, for every pixel in an image.

\subsection{Gas and Ice giants}

Cold planets emit relatively little thermal radiation and shine with reflected starlight at visible wavelengths. Flux and polarization spectra of this reflected starlight contain a wealth of information about an exoplanet atmospheric composition, structure, and possibly surface (for rocky exoplanets). The fluxes depend on the planet radius, orbital distance, and distance to the observer. Giant planets (both gaseous and iced) are definitely the main targets for SPICES since they will reflect more light than the emblematic but smaller Super Earths, so that a high level of atmospheric characterization can be achieved through both spectroscopy and polarimetry.

The depth and width of the spectral features yield information about overall atmospheric pressure and the existence and altitude of clouds and differ from one planet to the other (sometimes significantly) as shown in Fig. 1. With SPICES' spectral resolution of about 40, we will be able to identify various types of gases. The geometric albedos of Jupiter and Saturn are large at longer wavelengths because these planets have thick clouds relatively high in their atmospheres that efficiently reflect sunlight back to space. Shorter (bluer) wavelengths are absorbed by photochemical hazes instead of Rayleigh scattered. The geometric albedos of Uranus and Neptune are much lower but clearly show Rayleigh scattering at the shortest wavelengths, because for these colder planets, most of the cloud layers are located deeper in the atmosphere, which contain significantly more methane gas. Methane gas has many useful, measurable absorption bands of various strengths across SPICES spectral range. SPICES will also be able to characterize the atmospheric temperature and composition of a far greater variety of giant planets than found in our own solar system. As shown in Figure 1, warmer cloud-free atmospheres reflect less and thus have darker spectra, especially at longer (redder) wavelengths. Clouds increase the amount of reflected light (albedo) and also increase the contrast between continuum and adjoining strongly absorbing spectral regions. As temperature decreases for older or further-separated planets, the first clouds to appear in the skies are $\mathrm{H}_{2} \mathrm{O}$ water clouds. If temperatures get even colder the altitude of the $\mathrm{H}_{2} \mathrm{O}$ water clouds also gets lower and deeper into the atmosphere (since atmospheric temperature falls with 
altitude). Next, high-altitude $\mathrm{NH}_{3}$ ammonia clouds will form (with spectral features at $0.55,0.65$, and $0.79 \mu \mathrm{m}$ that are not shown in these plots), followed by the formation of $\mathrm{CH}_{4}$ methane clouds (strong features in 0.7-0.9 $\mu \mathrm{m}$ as shown in Fig. 1). Thus, the water and ammonia clouds that will likely be found in the atmospheres of planets in SPICES detection range will indicate atmospheric temperature and composition (Cahoy et al. (2010)).

\subsection{Super Earths}

Super Earths are very interesting objects but obviously fainter than giants. The largest terrestrial planets in the Solar System (Venus, Earth, Mars) differ strongly in composition, surface pressure, cloud coverage, and surface type at visible wavelengths. The Chappuis band of ozone $\mathrm{O}_{3}(590 \pm 50 \mathrm{~nm})$ is a prominent broad feature as well as the oxygen $\mathrm{O}_{2}$ A band $(760 \mathrm{~nm} \pm 10 \mathrm{~nm})$ and various water $\mathrm{H}_{2} \mathrm{O}$ bands. In favorable circumstances, SPICES could detect the most obvious spectral features (Maire et al. this proceeding) and polarization effects providing the degree of polarization is large like for Titan. SPICES can make the difference between various types of surfaces (forests / oceans) with very strong color effects in the visible as long as the atmosphere is optically thin. Observations of a planet at different locations in its orbit could reveal seasonal effects provided they are strong, as they might be for a planet in a highly elliptical orbit.

\section{Instrumental concept}

For the purpose of thermal stability, target accessibility, and high data rate for the full mission, SPICES will be on an orbit around the Sun-Earth L2 Lagrangian point. The payload concept is designed to fulfill the science requirements given above. The general problem to directly image extrasolar planets near bright stars is well known and emblematic techniques like coronagraphy and wavefront control have been developed over the last 15 years to enable high contrast. SPICES is a 1.5-m off-axis telescope consisting of a coronagraphic system combined with wavefront correction and feeding an integral spectro-polarimeter to cover the 450-900 $\mathrm{nm}$ band and measure two linear polarizations $(\mathrm{Q}, \mathrm{U})$. The optical quality of the telescope is not drastic (typically $10 \mathrm{~nm} \mathrm{rms}$ at mid frequencies on the primary). The wavefront control is achieved with the Electric Field Conjugation (Give'on et al. 2007), a specific algorithm to measure the aberrations, and a deformable mirror (DM) to correct the wavefront, providing a quality/stability on the order of tens of picometers. Our goal is to implement a more promising technique, the Self Coherent Camera (Galicher et al. 2008), which for now has a lower Technology Readiness Level but will allow a better discrimination of planets and speckles (Baudoz et al. 2006). We chose the Vector Vortex Coronagraph (VVC), a derivation of the phase mask concept which can be made potentially achromatic on a $50 \%$ bandwidth (Mawet et al. 2009). The backend instrument is a microlenses based integral field spectrograph (IFS) similar to those developed now on the ground for SPHERE and GPI (Antichi et al. 2009). Polarimetry is intimately implemented in the design by using a rotating half-wave retarder as a modulator and a polarizing beam-splitter cube as an analyzer. A detailed description of the instrumental concept can be found in Boccaletti et al. (2012).

\section{Expected performance}

A numerical model of the instrument was built to assess the performance of SPICES. We assume some realistic level of static wavefront errors $(20 \mathrm{~nm} \mathrm{rms}$ ), a $64 \times 64 \mathrm{DM}$, a VVC (achromatic), and the use of a self-coherent camera (SCC). The simulation iterates 

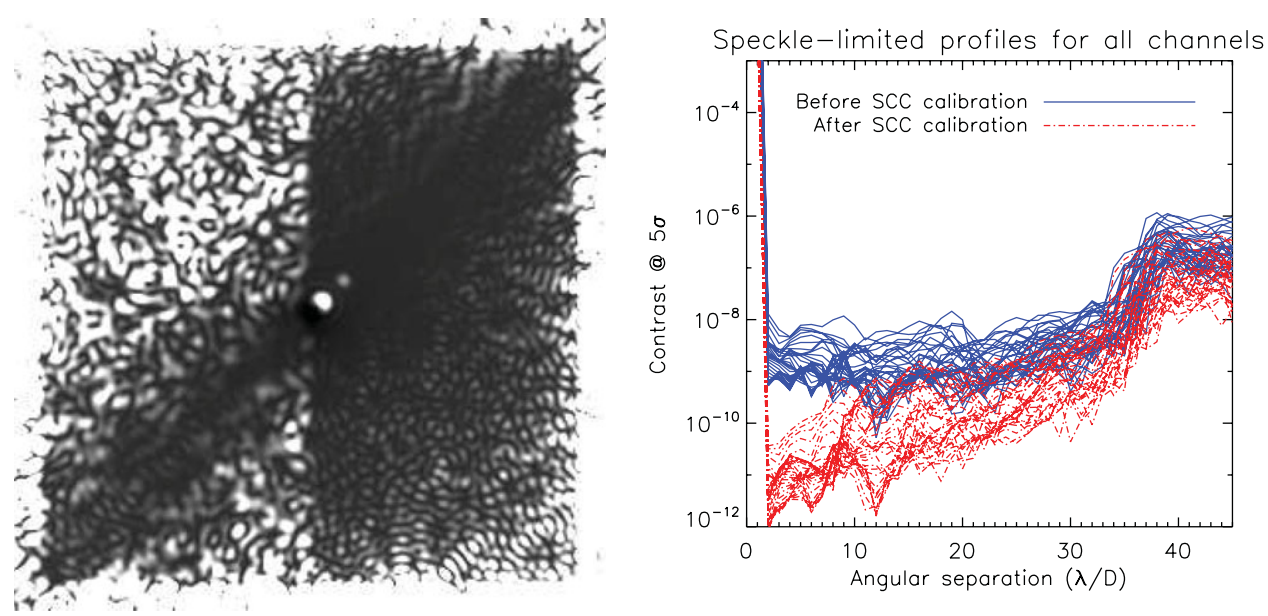

Figure 2. Left: simulated images of SPICES in one spectral channel $(\lambda=675 \mathrm{~nm})$ showing the $64 \times 64 \lambda / D$ corrected field of view where two fake planets at $10^{-8}$ and $10^{-9}$ contrast were added. Right: Corresponding 5-sigma contrast curves of SPICES for all spectral channels, with wavefront control alone (blue) then with an additional speckle calibration technique (red).

on the wavelengths (35 channels with the resolution ranging from 35 to 70 ) to produce $(x, y, \lambda)$ data cubes as the final product of the instrument (we did not account for polarimetry at this stage). As the SCC corrects for speckles inside the region covered by the DM actuators, the achievable contrast is limited by the intensity of the speckles outside this region. The SCC measures the wavefront in the focal plane, drives the DM, and finally improves the image quality by post-processing. The sampling is three pixels at the shortest wavelength. We also assume imperfection of flat field $(0.5 \%)$ and read-out-noise of $0.2 \mathrm{e}-/$ pix with a full-well capacity of $300 \mathrm{ke}-/ \mathrm{pix}$.

Figure 2 shows the contrast curves without noise obtained with a setup combining a DM and a coronagraph (blue curves) and the same setup plus a SCC post-processing (red curves) that improves the speckle rejection by two orders of magnitude in some spectral channels. We satisfy the requirement of a few $10^{-9}$ contrast at $2 \lambda / \mathrm{D}$ while the post-processing speckle calibration is definitely mandatory to achieve $10^{-10}$ contrasts appropriate for the characterization of Super-Earths. The contrast at short separation is limited by the inner working angle of the coronagraph (about $2 \lambda / \mathrm{D}$ ) and at large separation by the outer working angle of the wavefront control $(32 \lambda / \mathrm{D})$.

Using these contrast curves together with detection noise model, we first explored the performance in terms of detection $(S N R \geqslant 5)$ for several types of stars. Figure 3 shows the lambda-averaged achievable contrasts for G2 and M0 stars located at 3, 10 and 20 pc. For a set of typical planets (Jupiter-like, Neptune-like and Super Earths), and assuming a maximum integration time of 200 hours, we determined the maximal distances and optimal separations at which SPICES can perform detection. Table 1 draws the conclusion about this parameter space. We estimated a sample of about 300 stars within $20 \mathrm{pc}$ for which SPICES can search for planets between 0.5-10 AU.

The main objective of SPICES is the spectro-polarimetric characterization of already known planets. Using our instrumental model combined with atmospheric models from Cahoy et al. (2010), and Stam (2008) we performed simulations of SPICES spectrometric capabilities. In the prospect of comparative exo-planetology, we first derived which $S N R$ is required to differentiate between two exoplanet models where one particular parameter has changed (for instance the metallicity). As for illustration, we simulated two cases for 
Table 1. Range of physical separations (in AU) achievable with SPICES for $S N R \geqslant 5$ for three stellar types and three categories of planets (Jupiter, Neptune, Super Earths). The maximal distance of the stars are also indicated.

\begin{tabular}{llll}
\hline & \multicolumn{3}{c}{ separation range [AU] } \\
& Jupiter & Neptune & Super Earth \\
\hline $\mathrm{A} 0<20 \mathrm{pc}$ & $1-10$ & $1-3$ & $1-3$ \\
$\mathrm{G} 2<10 \mathrm{pc}$ & $1-6$ & $1-3$ & $1-2$ \\
$\mathrm{M} 0<7.5 \mathrm{pc}$ & $0.5-4$ & $0.5-1.5$ & $0.5-1$ \\
\hline
\end{tabular}
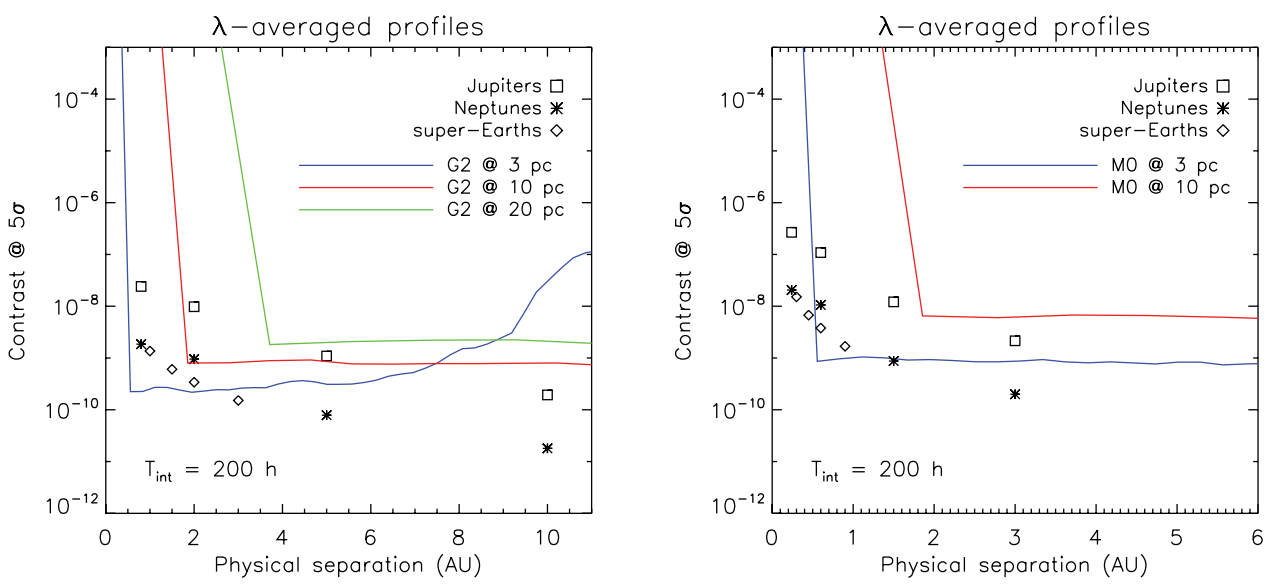

Figure 3. Contrast at 5 sigma obtained for G2 (left) and M0 (right) stars at different distances (colored curves). Symbols correspond to the expected intensities of some typical exoplanets as derived from Cahoy et al. (2010) and Stam (2008) (squares for Jupiters, stars for Neptunes and diamonds for Super Earths).

Jupiter-like and Neptune-like planets from which we can address two particular questions from the observational point of view. The first question is: if a giant planet is on an eccentric orbit can we measure a temporal variation of its atmosphere? The second question is: if two iced giants differ in metallicity can we actually see this difference?

For the first case we consider a Jupiter-like planet at two separations, 0.8 and 2 AU from a G2V star (for instance corresponding to periastron/apoastron, $e=0.43$ ). The closest separation of $0.8 \mathrm{AU}$ imposes a maximum star distance of $4 \mathrm{pc}$ accessible with SPICES angular resolution. From the model of Cahoy et al. (2010) we expect some differences due to the formation of clouds following the planet temperature. In Fig. 4 (left) the two spectra measured in the simulated image are clearly disentangled especially in the blue where the closest model has its atmosphere dominated by Rayleigh scattering. Methane bands at $0.73 \mu \mathrm{m}$ and water bands at $0.82 \mu \mathrm{m}$ are also weaker when the planet gets colder. These differences are detectable with SPICES at $S N R=15$ in only 30 minutes of integration time.

The second case deals with the capability to distinguish two Neptune-like planets of different metallicities (here $10 \times$ and $30 \times$ solar) but located at the same separation (2 AU). Since these planets are fainter than Jupiter-like planets and metallicity requires a higher $S N R$, a much longer integration time is requested (100 hours to achieve $S N R=$ $25)$ but again the differences are detected in the methane and water bands which are deeper when the metallicity is higher. The extension of this second example also indicates that SPICES has the ability to distinguish between gas and ice giants if they differ in 

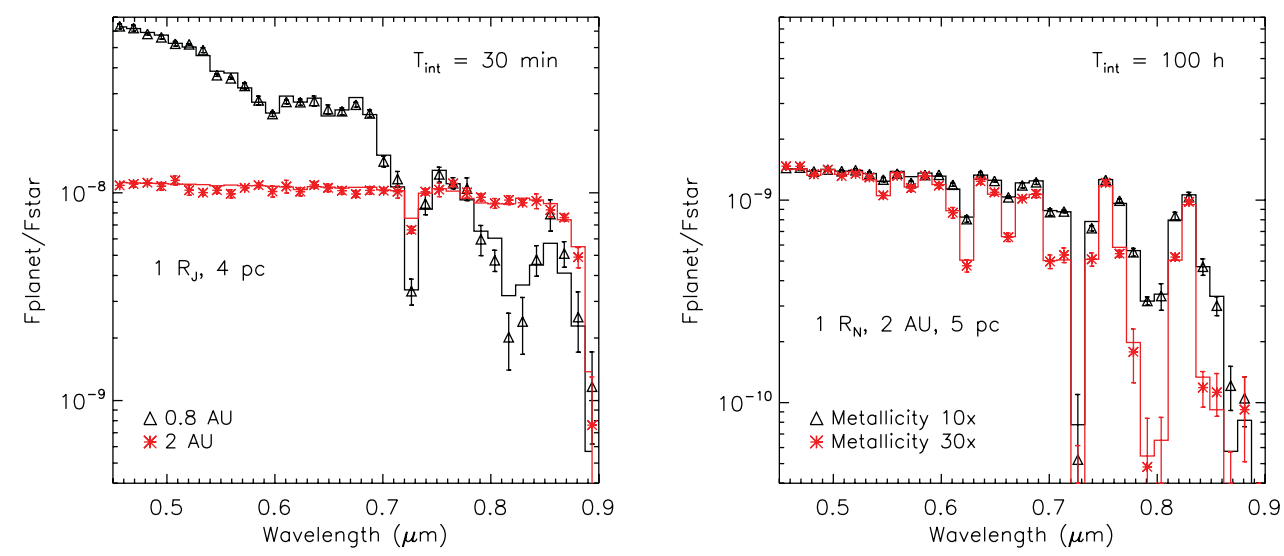

Figure 4. Simulated spectra across the SPICES spectral range, of two gas giant planets (left) located at respectively 0.8 and $2 \mathrm{AU}$, and two ice giant planets with different metallicities. Error bars are shown at 1-sigma. The main spectral features correspond to water and methane bands.

metallicity like in the Solar System. Several other simulations were presented in Maire et al. (2012) to assess the performance of SPICES for characterizing extrasolar planets (see Maire et al. this proceeding for the case of Super Earths).

\section{Conclusion}

In the context of high contrast imaging from space, several critical sub-systems are being developed (wavefront control, coronagraphy) in order to reach a technological readiness level adequate for a space mission. In parallel, the numerical simulation activity presented in Maire et al. (2012) has shown the ability of a mission like SPICES to measure the atmospheric properties of giants as well as Super Earths in a volume of about $20 \mathrm{pc}$ and for separation in the 0.5-10 AU range, precisely where most exoplanets are expected to reside according to the core accretion formation model. Therefore, SPICES will be a mandatory tool for comparative exo-planetology of long-period planets and will complement other direct detection programs, especially the spectroscopy of close-in transiting planets achievable from transit spectroscopy.

\section{References}

Antichi, J., Dohlen, K., Gratton, R. G., et al. 2009, ApJ, 695, 1042

Baudoz, P., Boccaletti, A., Baudrand, J., \& Rouan, D. 2006, IAU Colloq. 200, 553

Boccaletti, A., Schneider, J., Wes, T., et al. 2012, Experimental Astronomy, 34, 355

Cahoy, K. L., Marley, M. S., \& Fortney, J. J. 2010, ApJ, 724, 189

Galicher, R., Baudoz, P., \& Rousset, G. 2008, A\&A, 488, L9-L12

Give'on, A., Kern, B., Shaklan, S., et al. 2007, SPIE Conf. Series, 6691

Karkoschka, E. 1994 Icarus, 111, 174

Levine, M., Lisman, D., Shaklan, S., et al. 2009, arXiv, 0911.3200

Maire, A.-L., Galicher, R., Boccaletti, A., et al. 2012, A\& A, 541, A83

Mawet, D., Trauger, J. T., Serabyn, E., et al. 2009, SPIE Conf. Series, 7440

Schneider, J., Boccaletti, A., Mawet, D., et al. 2009, Experimental Astronomy, 23, 357

Stam, D. M. 2008, A\&3A, 482, 989 